\title{
Diffraction at the Tevetron: CDF Results
}

\author{
Konstantin Goulianos*i \\ The Rockefeller University, 1230 York Avenue, New York, NY 10023, USA \\ E-mail: dino@mail.rockefeller.edu
}

\begin{abstract}
The diffractive program of the CDF Collaboration at the Fermilab Tevatron $p \bar{p}$ Collider is reviewed with emphasis on recent results from Run II at $\sqrt{s}=1.96 \mathrm{TeV}$. Updated results on the $x_{B j}$ and $Q^{2}$ dependence of the diffractive structure function obtained from dijet production, and on the slope parameter of the $t$-distribution of diffractive events as a function of $Q^{2}$ in the range $1 \mathrm{GeV}^{2}<Q^{2}<10^{4} \mathrm{GeV}^{2}$, are presented and compared with theoretical expectations. Results on cross sections for exclusive dijet and diphoton production are also presented and used to calibrate theoretical estimates for exclusive Higgs production at the Large Hadron Collider.
\end{abstract}

DIFFRACTION 2006 - International Workshop on Diffraction in High-Energy Physics

September 5-10 2006

Adamantas, Milos island, Greece

\footnotetext{
* Speaker.

${ }^{\dagger}$ Presented on behalf of the CDF collaboration.
} 


\section{Introduction}

The CDF collaboration has been involved in a systematic and comprehensive program of studies of diffractive interactions since the start of operations of the Fermilab Tevatron $p \bar{p}$ collider in 1989. The ultimate goal of this program is to provide experimental results which will be of help in elucidating the QCD character of hadronic diffraction [1]. Diffractive interactions are characterized by large rapidity gaps ${ }^{1}$ in the final state, presumed to occur via the exchange of a quark/gluon combination carrying the quantum numbers of the vacuum. This exchange is traditionally referred to as Pomeron [2]. The process which is directly analogous to the classical diffraction of light is elastic scattering, but it is inelastic diffraction processes that provide the most stringent tests for QCD inspired models of diffraction. The total cross section is also of interest in testing theoretical models of diffraction, since it is related to the imaginary part of the forward elastic scattering amplitude through the optical theorem. In this paper, we present results obtained at the Tevatron by $\mathrm{CDF}$ and comment on their physics significance.

The names / dates of the Tevatron runs and integrated luminosities of data collected by CDF are listed below:

\begin{tabular}{llr} 
Run Number & Date & $\int \operatorname{Lum}\left(\mathrm{pb}^{-1}\right)$ \\
\hline$\underline{\text { Run I }}$ & & \\
IØ & $1988-1989$ & 5 \\
Ia & $1992-1993$ & 20 \\
Ib & $1993-1995$ & 100 \\
Ic & $1995-1996$ & 10 \\
$\underline{\text { Run II }}$ & & \\
IIa & $2003-2006$ & 1000 \\
IIb & currently in progress &
\end{tabular}

In Run IØ, CDF measured elastic, single diffractive, and total cross sections at $\sqrt{s}=630$ and $1800 \mathrm{GeV}$.

In Runs Ia, Ib and Ic, CDF studied both soft and hard diffractive processes, with the latter incorporating a hard partonic scattering in addition to the characteristic large rapidity gap of diffraction. Figure 1 shows schematically the diagrams and final state event topologies of the processes studied by CDF in Run I.

In Run II, the CDF diffractive program was enhanced by extending the kinematic range of the measurements of hard diffractive processes and by additional studies of exclusive production processes.

All Run I results have been published. These results are briefly summarized. The Run II results are discussed in more detail. Recent results on the $x$-Bjorken and $Q^{2}$ dependence of the diffractive structure function and on the $t$-dependence of diffractive cross sections are reported and characterized in terms of their physics content. In addition, results on exclusive dijet and diphoton production are presented and their significance in calibrating predictions for exclusive Higgs boson production at the LHC is discussed.

\footnotetext{
${ }^{1}$ Rapidity gaps are regions of rapidity devoid of particles; rapidity, $y=\frac{1}{2} \frac{E+p_{L}}{E-p_{L}}$, and pseudorapidity, $\eta=-\ln \tan \frac{\theta}{2}$, are used interchangeably, as in the kinematic region of interest the values of these two variables are approximately equal.
} 


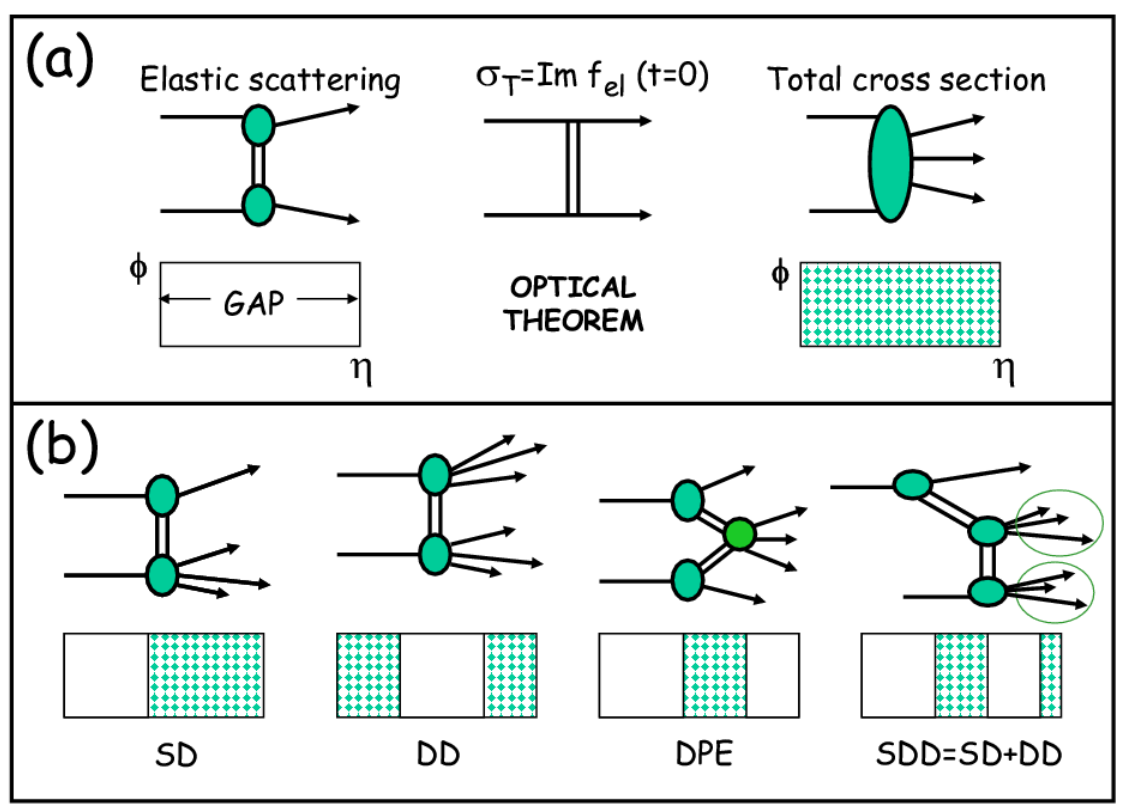

Figure 1: Schematic diagrams and event topologies in azimuthal angle $\phi$ versus pseudorapidity $\eta$ for (a) elastic and total cross sections, and (b) single diffraction (SD), double diffraction (DD), double Pomeron exchange (DPE), and double plus single diffraction cross sections (SDD=SD+DD). The hatched areas represent regions in which there is particle production.

\section{Run IØ Results}

In Run IØ, CDF measured the elastic, soft single diffractive, and total $p \bar{p}$ cross sections at $\sqrt{s}=630$ and $1800 \mathrm{GeV}$. The measurement was performed with the CDF I detector, which during run IØ had tracking coverage out to $|\eta| \sim 7$ and Roman Pot Spectrometers on both sides of the Interaction Point (IP). The normalization was obtained by the luminosity independent method, which is based on simultaneously measuring the total interaction rate, which depends on $\sigma_{T}$, and the elastic scattering differential rate at $t=0$, which depends on $\sigma_{T}^{2}$ (optical theorem):

$$
\left.\sigma_{T} \propto \frac{1}{L}\left(N_{e l}+N_{\text {inel }}\right) \quad \& \quad \sigma_{T}^{2} \sim \frac{1}{1+\rho^{2}} \frac{d N_{e l}}{d t}\right|_{t=0} \Rightarrow \sigma_{T}=\left.\frac{16 \pi}{1+\rho^{2}} \frac{1}{N_{e l}+N_{\text {inel }}} \frac{d N_{e l}}{d t}\right|_{t=0}
$$

Paradoxically, overestimating the total rate, as for example due to background events, yields smaller elastic and total cross sections, while loss of inelastic events results in larger cross sections.

Figure 2 (left) shows Regge based fits to total and elastic scattering data using the eikonal approach to ensure unitarity [3]. Good fits are obtained, which are consistent with the CDF cross sections at the Tevatron even if the Tevatron cross sections are not used in the fit [3]. In contrast, the standard Regge fit to total single diffractive cross sections, shown in Fig. 目 (right), overestimates the Tevatron cross sections by a factor of $\sim 10$. This discrepancy represents a breakdown of factorization, which is restored by the renormalization procedure proposed in Ref. [沛. 

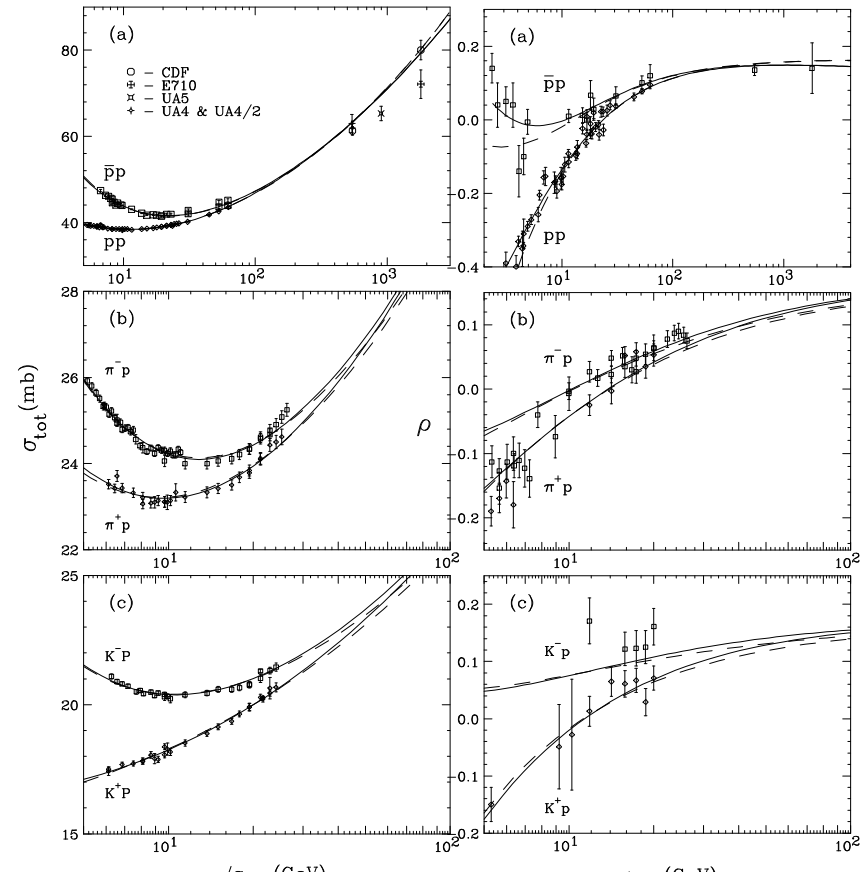

$\sqrt{\mathrm{s}} \quad(\mathrm{GeV})$

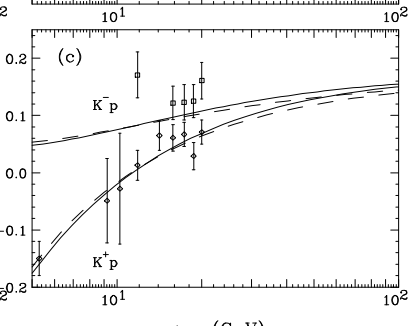

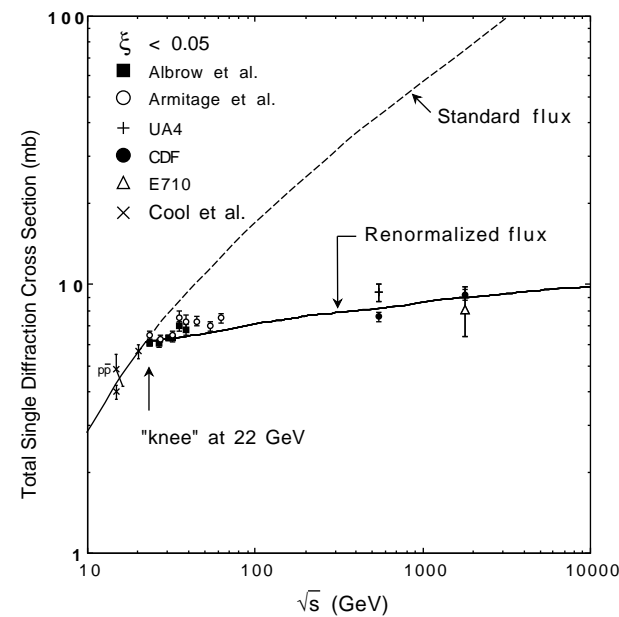

Figure 2: (left) Simultaneous fit to $p \bar{p}, \pi^{ \pm}$, and $K^{ \pm}$total cross section and $\rho$-value data using eikonalized (solid) and Born level (dashed) amplitudes [3] - the rise of the $p \bar{p}$ cross section with $\sqrt{s}$ is "pulled" by the rise of the $\pi^{ \pm}$cross sections and would pass through the CDF point at $\sqrt{s}=1800 \mathrm{GeV}$ even if this point were not used in the fit; (right) total $p p / p \bar{p}$ single diffraction dissociation cross section data (sum of $\bar{p}$ and $p$ dissociation) for $\xi<0.05$ compared with Regge predictions based on standard and renormalized Pomeron flux [ [†].

\section{Run Ia,b,c Results}

The diffractive processes studied by CDF in Tevatron Runs Ia,b,c (1992-1996) are schematically shown in Fig. 1 $\mathrm{b}$. Both soft and hard processes were studied. A discussion of the results obtained and of their significance in deciphering the QCD nature of the diffractive exchange can be found in Ref. [5]. The most interesting discoveries from this diffractive program were the breakdown of factorization and the restoration of factorization in events with multiple rapidity gaps.

Breakdown of factorization. At $\sqrt{s}=1800 \mathrm{GeV}$, the SD/ND ratios (gap fractions) for dijet, $W$, $b$-quark, and $J / \psi$ production, as well the ratio of $\mathrm{DD} / \mathrm{ND}$ dijet production, are all $\approx 1 \%$. This represents a suppression of a factor of $\sim 10$ relative to predictions based on diffractive parton densities measured from DDIS at HERA, indicating a breakdown of QCD factorization comparable to that observed in soft diffraction processes relative to Regge theory expectations. However, factorization approximately holds among the four different diffractive processes at fixed $\sqrt{s}$, which indicates that the suppression has to do with the formation of the rapidity gap, as predicted by the generalized gap renormalization model (see [5]).

Restoration of factorization in multi-gap diffraction. Another interesting aspect of the data is that ratios of two-gap to one-gap cross sections for both soft and hard processes obey factorization. This provides both a clue to understanding diffraction in terms of a composite Pomeron and an experimental tool for diffractive studies using processes with multiple rapidity gaps (see [阿). 


\section{The Run II Diffractive Program}

In Run II, CDF has been conducting the following studies of diffraction:

- structure function in dijet production,

$-t$ distributions,

- exclusive dijet, diphoton, and $e^{+} e^{-}$production,

- structure function in $W$ production,

- gap between jets: dependence of the cross section on gap size for fixed $\Delta \eta^{j e t}$.

In this paper, we present preliminary results on the first three topics. The diffractive $W$ and 'gap between jets' analyses are in progress and results are expected in early 2007.

\subsection{Run II forward detectors}

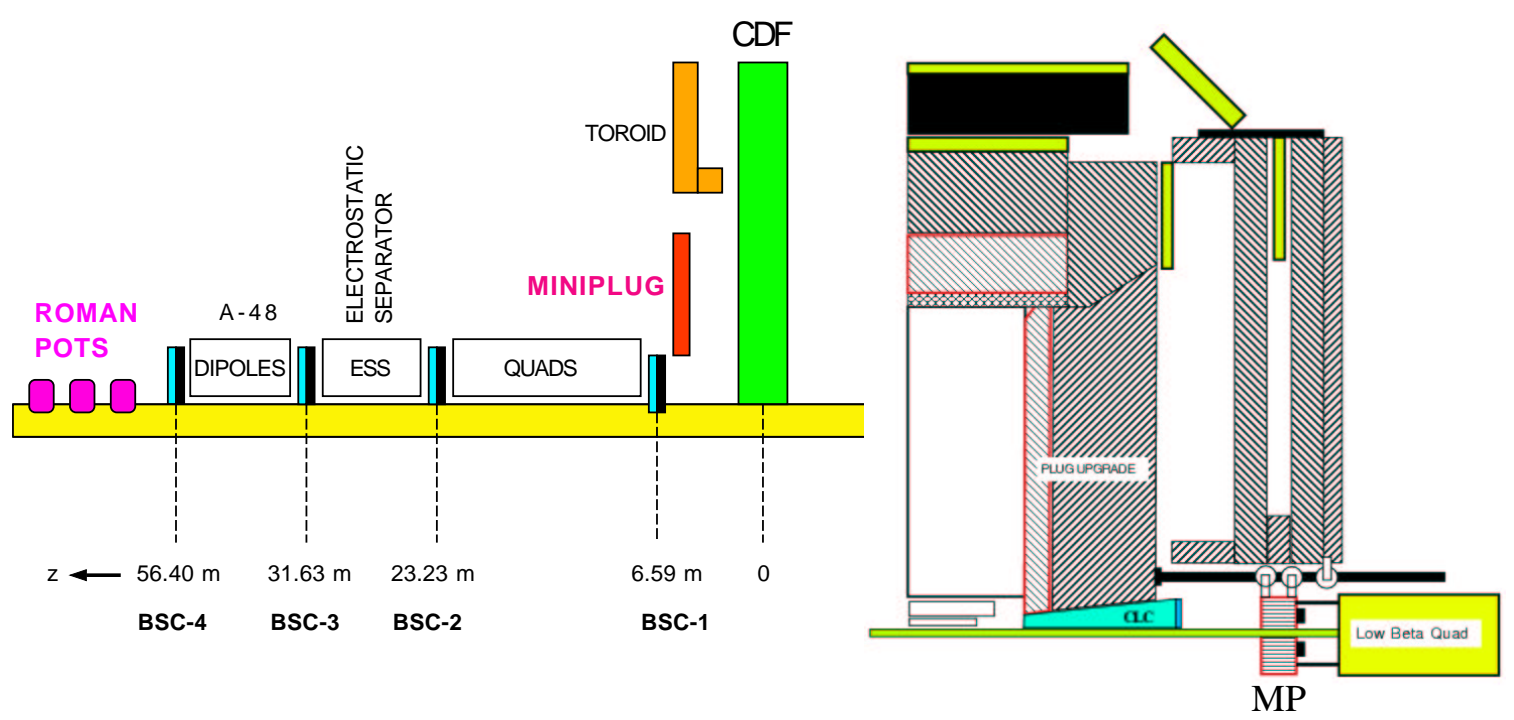

Figure 3: The CDF detector in Run II: (left) location of forward detectors along the $\bar{p}$ direction; (right) position of the Cerenkov Luminosity Monitor (CLC) and MiniPlug calorimeters (MP) in the central detector.

The Run II diffractive program was made possible by an upgraded CDF detector [6], which includes the following special forward components (Fig. 3):

- Roman Pot Spectrometer (RPS) to detect leading antiprotons,

- MiniPlug (MP) forward calorimeters approximately covering the region $3.5<|\eta|<5.5$,

- Beam Shower Counters (BSC) positioned around the beam pipe at four (three) locations along the $\bar{p}(p)$ beam direction to tag rapidity gaps within $5.5<|\eta|<7.5$.

The Roman Pot Spectrometer is the same one that was used in Run Ic. It consists of $X-Y$ scintillation fiber detectors placed in three Roman Pot Stations located at an average distance of $57 \mathrm{~m}$ downstream in the $\bar{p}$ direction. The detectors have a position resolution of $\pm 100 \mu \mathrm{m}$, which makes possible a $\sim 0.1 \%$ measurement of the $\bar{p}$ momentum. In Run Ic, the $\bar{p}$-beam was behind the proton beam, as viewed from the RPS side. An inverted polarity (with respect to Run I) of the electrostatic beam separators enabled moving the RPS detectors closer to the $\bar{p}$-beam and thereby gain acceptance for small $|t|$ down to $\xi \equiv 1-x_{F}(\bar{p})=0.03$ (for larger $|t|$, lower $\xi$ values can be reached). 
The MiniPlug calorimeters are placed within the holes of the muon toroids. They consist of layers of lead plates immersed in liquid scintillator. The scintillation light is picked up by wavelength shifting fibers strung through holes in the lead plates and read out by multi-channel PMT's. The calorimeter "tower" structure is defined by arranging fibers in groups to be read out by individual PMT pixels. There are 84 towers in each MiniPlug, and the signals they provide can be used to measure energy and position for both electromagnetic and hadron initiated showers [7].

The Beam Shower counters are rings of scintillation counters "hugging" the beam pipe. The BSC-1 rings are segmented into four quadrants, while in the other BSCs are segmented into two halves. The BSC-1 are also used to provide rapidity gap triggers and for measuring beam losses.

\subsection{Diffractive structure function from dijet production}

In Run II, CDF has obtained preliminary results for the $x_{B j}, Q^{2}$, and $t$ dependence of the diffractive structure function from dijet production at $\sqrt{s}=1960 \mathrm{GeV}$. The measured $x_{B j}$ rates confirm the factorization breakdown observed in Run I (see review in Ref. [8]). The $Q^{2}$ and $t$ dependence results are shown in Fig 4
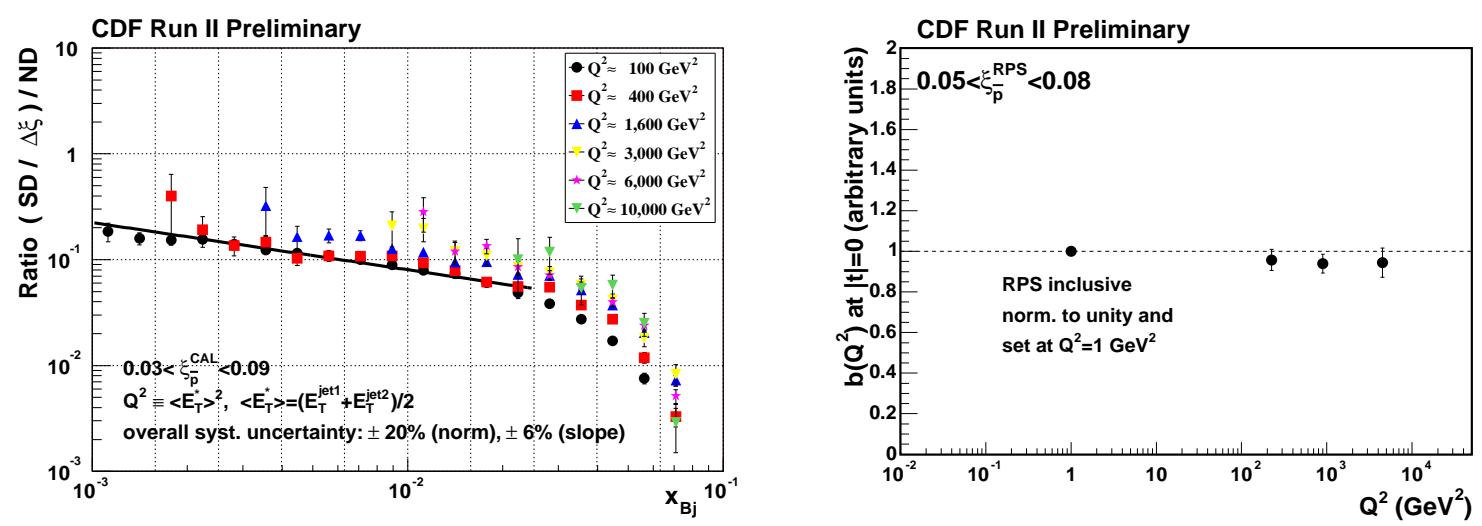

Figure 4: (left) Ratio of diffractive to non-diffractive dijet event rates as a function of $x_{B j}$ (momentum fraction of parton in antiproton) for different values of $E_{T}^{2}=Q^{2} ;\left.($ right $) b\right|_{t=0}$ slope vs $Q^{2}$.

$Q^{2}$ dependence. In the range $10^{2} \mathrm{GeV}^{2}<Q^{2}<10^{4} \mathrm{GeV}^{2}$, where the inclusive $E_{T}$ distribution falls by a factor of $\sim 10^{4}$, the ratio of the SD/ND distribution increases, but only by a factor of $\sim 2$. This result indicates that the $Q^{2}$ evolution in diffractive interactions is similar to that in ND interactions.

$t$-dependence. The slope parameter $\left.b\left(Q^{2}\right)\right|_{t=0}$ of an exponential fit to $t$ distributions near $t=0$ shows no $Q^{2}$ dependence in the range $1 \mathrm{GeV}^{2}<Q^{2}<10^{4} \mathrm{GeV}^{2}$.

These results support the picture of a composite Pomeron formed from color singlet combinations of the underlying parton densities of the nucleon (see [5]).

\subsection{Exclusive Dijet Production}

Exclusive production in $p \bar{p}$ collisions is of interest not only for testing QCD inspired models of diffraction, but also as a tool for discovering new physics. The process that has attracted the 
most attention is exclusive Higgs boson production. The search for Higgs bosons is among the top priorities in the research plans of the LHC experiments. While the main effort is directed toward searches for inclusively produced Higgs bosons, an intense interest has developed toward exclusive Higgs production, $\bar{p} / p+p \rightarrow \bar{p} / p+H+p$. This Higgs production channel presents several advantages: it can provide clean events in an environment of suppressed QCD background, in which the Higgs mass can accurately be measured using the missing mass technique by detecting and measuring the momentum of the outgoing proton and (anti)proton. However, exclusive production is hampered by expected low production rates [9]. As rate calculations are model dependent and generally involve non-perturbative suppression factor(s), it is considered prudent to calibrate them against processes involving the same suppression factors(s), but have higher production rates that can be measured at the Tevatron. One such processes is exclusive dijet production, which proceeds through the same mechanism as Higgs production, as shown in Fig. 5 .
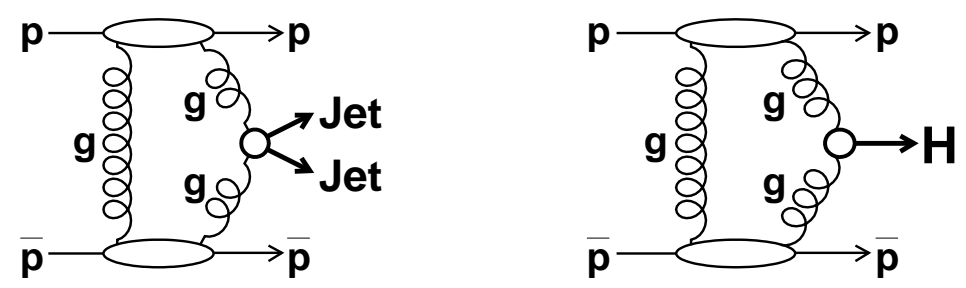

Figure 5: Lowest order diagrams for exclusive dijet (left) and Higgs (right) production in $\bar{p} p$ collisions.

The search for exclusive dijets is based on measuring the dijet mass fraction, $R_{j j}$, defined as the mass of the two leading jets in an event, $M_{j j}$, divided by the total mass reconstructed from the energy deposited in all calorimeter towers, $M_{X}$. The signal from exclusive diets is expected to appear at high values of $R_{j j}$, smeared by resolution and radiation effects. Events from inclusive DPE production, $\bar{p} p \rightarrow \bar{p}+g a p+j j+X+g a p$, are expected to contribute to the entire $M_{j j}$ region. Any such events within the exclusive $M_{j j}$ range contribute to background and must be subtracted when evaluating exclusive production rates.

The method used to extract the exclusive signal from the inclusive $R_{j j}$ distribution is based on fitting the data with MC simulations [10]. Two methods have been used. In the first one, the POMWIG and ExHuME generators are used for simulating inclusive and exclusive events, respectively; in the second, inclusive (exclusive) distributions are simulated using the POMWIG (DPEMC) program. Experimentally, the MC non-exclusive dijet background shape is checked by a study of high $E_{T} b$-tagged dijet events, as quark jet production through $g g \rightarrow \bar{q} q$ is suppressed in LO QCD by the $J_{z}=0$ selection rule as $m_{q} / M^{\text {jet }} \rightarrow 0$.

Figure 6 shows measured $R_{j j}$ distributions plotted versus dijet mass fraction. On the left, the number of events within the specified kinematic region is compared with fits based on POMWIG plus ExHuME distribution shapes, and on the right with fits based on POMWIG $\oplus$ DPEMC predictions. Both approaches yield good fits to the data. The suppression factor expected for exclusive $b$-tagged dijet events is checked with CDF data in Fig. 7. Within the quoted errors, this result validates the MC based method for extracting the exclusive signal. 

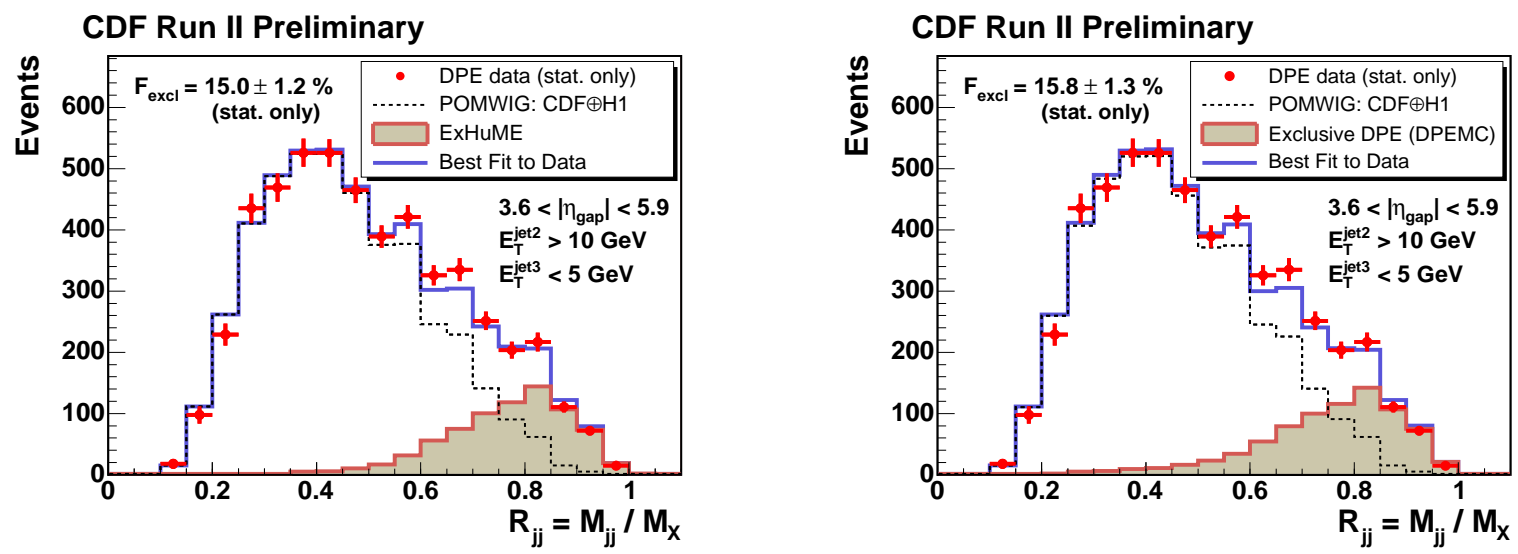

Figure 6: Extraction of exclusive dijet production signal using Monte Carlo techniques to subtract the inclusive dijet background: (left) dijet mass fraction in data (points) and best fit (solid line) obtained from MC events generated using the POMWIG (dashed) and ExHuME (filled) MC generators for inclusive and exclusive events, respectively; (right) the same data fitted with POMWIG and exclusive DPEMC generators.

\section{CDF Run II Preliminary}

ᄂ

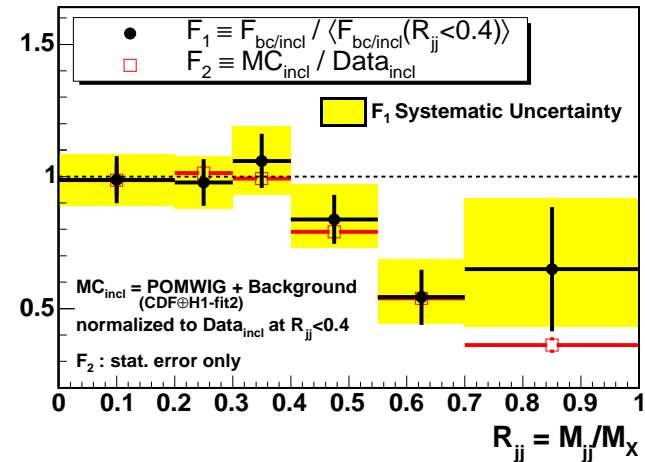

Figure 7: (circles) Fraction of heavy flavor $(b, c)$ to all dijet events in data, $F_{1}$, as a function of dijet mass fraction showing the expected suppression at high $M_{j j}$; (squares) fraction, $F_{2}$, of inclusive MC to data from Fig. 6 (left). The agreement between the measured suppression levels in $F_{1}$ and $F_{2}$ serves to validate the MC based technique for extracting the exclusive production rate from the data.

\section{CDF Run II Preliminary}

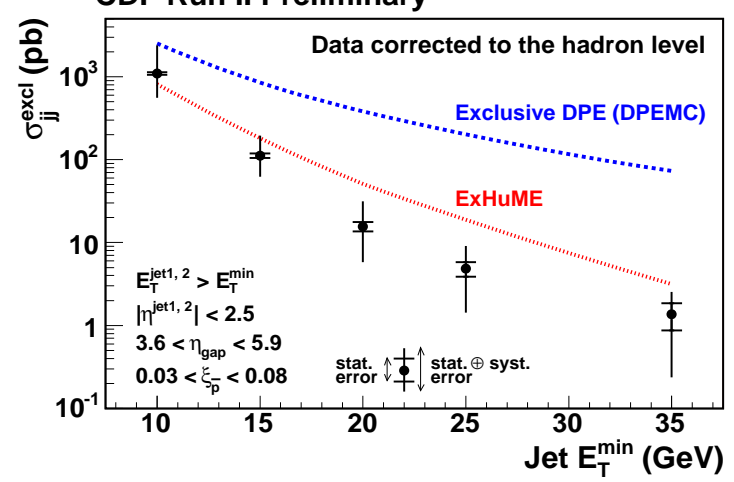

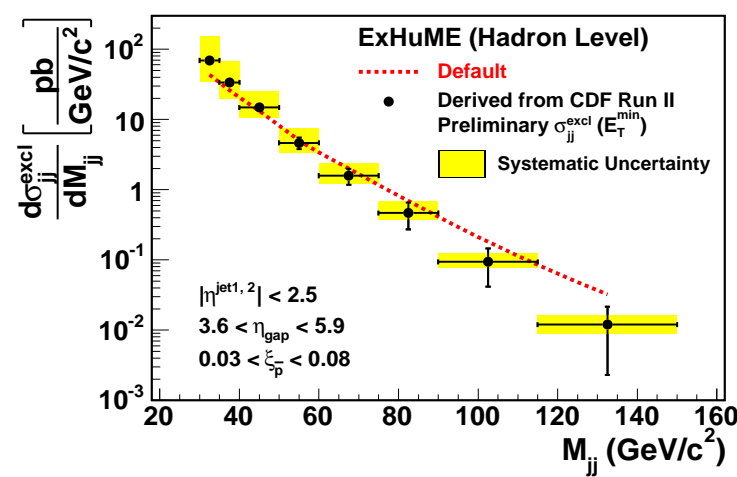

Figure 8: (left) Measured exclusive dijet cross sections versus the minimum $E_{T}$ of the two leading jets compared with ExHuME and DPEMC predictions; (right) ExHuME hadron level differential exclusive dijet cross section vsersus dijet mass normalized to the CDF cross sections at left. The systematic errors shown are propagated from those in the data; the ExHuME predictions have comparable systematic uncertainties. 
In Fig. 8 (left), integrated cross sections above a minimum $E_{T}^{\text {jet } 1,2}$ are compared with ExHuME and DPEMC predictions. The data favor the ExHuME prediction. ExHuME hadron level differential cross sections $d \sigma^{\text {excl }} / d M_{j j}$ normalized to the measured data points of Fig. 8 (left) are shown in Fig. 8 (right) with errors propagated from the uncertainties in the data. Within the errors, the good agreement with the default ExHuME prediction up to masses in the region of the standard model Higgs mass predicted from global fits to electroweak data lends credence to the calculation of Ref. [9]. for exclusive Higgs boson production at the LHC.

\subsection{Exclusive $\gamma \gamma$ production}

Exclusive $\gamma \gamma$ production in $p \bar{p}$ collisions proceeds through a lowest order diagram similar to that of Fig. 5 (right), but with the gluons that produce the Higgs replaced by $\gamma$ 's. Therefore, like exclusive dijet production, exclusive $\gamma \gamma$ production can also be used for calibrating models of Higgs production at hadron colliders.

A search for exclusive $\gamma \gamma$ production has been performed on a sample of events collected by requiring a high $E_{T}$ electromagnetic shower in combination with a loose forward rapidity gap requirement. In the data analysis, the rapidity gap requirement was tightened, and the search was narrowed down to events with two high $E_{T}$ photon showers satisfying certain "exclusivity" requirements. In a data sample of $532 \mathrm{pb}^{-1}$ total integrated luminosity, three exclusive $\gamma \gamma$ candidate events with $E_{T}^{\gamma}>5 \mathrm{GeV}$ were found with no tracks pointing to the electromagnetic clusters. As a check of the robustness of the rapidity gap requirement, CDF measured the cross section for the purely QED process $\bar{p}+p \rightarrow \bar{p}+e^{+} e^{-}+p$, whose cross section can be reliably calculated. Twelve exclusive $e^{+} e^{-}$candidate events were found in the data with an estimated background of $2.1_{-0.3}^{+0.7}$, yielding $\sigma(\gamma \gamma)=0.14_{-0.04}^{+0.14}$ (stat) \pm 0.03 (syst) pb and $\sigma\left(e^{+} e^{-}\right)=1.6_{-0.3}^{+0.5}$ (stat) \pm 0.3 (syst) pb, which agrees with an expectation of $1.711 \pm 0.008 \mathrm{pb}$. For $\gamma \gamma$ production, the background estimate is not yet complete; based on Ref. [9], the number of events expected in this data sample is $1_{-1}^{+3}$.

\section{Summary}

The diffractive program of the CDF Collaboration at the Fermilab Tevatron $p \bar{p}$ Collider has been reviewed with emphasis on recent results from Tevatron Run II at $\sqrt{s}=1.96 \mathrm{TeV}$.

Run I results have been briefly presented and their physics significance placed in perspective. Processes studied by CDF in Run I include elastic and total cross sections, soft diffractive cross sections with single and multiple rapidity gaps, and hard single diffractive production of dijet, $W$, $b$-quark, and $J / \psi$ production, as well as central dijet production in events with two forward rapidity gaps (double Pomeron exchange). The results obtained support a picture of universality of diffractive rapidity gap formation across soft and hard diffractive processes, which favors a composite over a particle-like Pomeron made up from color singlet quark and/or gluon combinations with vacuum quantum numbers.

Run II results on the $x_{B j}$ and $Q^{2}$ dependence of the diffractive structure function obtained from dijet production have been presented, as well as on the slope parameter of the $t$-distribution of diffractive events as a function of $Q^{2}$. In the range $10^{2} \mathrm{GeV}^{2}<Q^{2}<10^{4} \mathrm{GeV}^{2}$, where the inclusive $E_{T}$ distribution falls by a factor of $\sim 10^{4}$, the ratio of SD/ND distributions varies by only a factor of $\sim 2$, indicating that the $Q^{2}$ evolution in diffractive interactions is similar to that in ND 
ones; and the slope parameter $\left.b\left(Q^{2}\right)\right|_{t=0}$ of an exponential fit to $t$ distributions near $t=0$ in the range $1 \mathrm{GeV}^{2}<Q^{2}<10^{4} \mathrm{GeV}^{2}$ shows no $Q^{2}$ dependence. These results support a picture of a composite diffractive exchange (Pomeron) made up from the underlying parton densities of the nucleon.

Results on cross sections for exclusive dijet and diphoton production have also been presented and their significance for calibrating theoretical estimates for exclusive Higgs production at the Large Hadron Collider discussed. The exclusive dijet cross section was measured up to jet $E_{T}^{\min }$ of $35 \mathrm{GeV}$. When expressed as a function of dijet mass $M_{j j}$, cross sections up to masses of $M_{j j} \sim$ $120-140 \mathrm{GeV}$ are obtained, which are in the region of the standard model Higgs mass expected from global fits to electroweak data. A measurement of exclusive $\gamma \gamma$ production, a process which can also be used for calibrating Higgs production models, yielded three events corresponding to a cross section in the range of that predicted in Ref. [9].

\section{References}

[1] K. Goulianos, Diffractive and total cross sections at Tevatron and LHC, in Hadron Collider Physics Symposium 2006, May 22-26, Duke University, Durham, NC, USA.

[2] P. D. B. Collins, An Introduction to Regge Theory and High Energy Physics, Cambridge University Press (1977); V. Barone and E. Predazzi, High-Energy Particle Diffraction, Springer Press (2001); S. Donnachie, G. Dosch, O. Nachtmann, and P. Landshoff, Pomeron Physics and QCD, Cambridge University Press (2002).

[3] R. J. M. Covolan, J. Montanha, and K. Goulianos, A New Determination of the Soft Pomeron Intercept, Phys. Lett. B 389, 176 (1996).

[4] K. Goulianos, Renormalization of Hadronic Diffraction and the Structure of the Pomeron, Phys. Lett. B 358, 379 (1995); Erratum-ib. 363, 268 (1995).

[5] K. Goulianos, Hadronic Diffraction: Where do we Stand?, in La Thuile 2004, Results and Perspectives in Particle Physics, edited by M. Greco, Proc. of Les Rencontres de Physique de la Vallé d'Aoste, La Thuile, Aosta Valley, Italy, February 29 - March 6, 2004, pp. 251-274; e-Print Archive: hep-ph/0407035.

[6] R. Blair et al. (CDF Collaboration), The CDF II Detector: Technical Design Report, FERMILAB-Pub-96/390-E.

[7] K. Goulianos and S. Lami, Performance of a Prototype Position Sensitive Towerless Calorimeter, Nucl. Instrum. Meth. A 430, 34-47 (1999); K. Goulianos et al., The CDF MiniPlug calorimeters, Nucl. Instrum. Meth. A 496, 333-346 (2003).

[8] M. Gallinaro (for the CDF collaboration), Diffractive and exclusive measurements at CDF, Presented at 14th International Workshop on Deep Inelastic Scattering (DIS 2006), Tsukuba, Japan, 20-24 Apr 2006; e-Print Archive: hep-ex/0606024.

[9] V. Khoze, A. Kaidalov, A. Martin, M. Ryskin, and W. Stirling, Diffractive processes as a tool for searching for new physics, e-Print Archive:hep-ph/0507040, and references therein.

[10] Monte Carlo programs used in the CDF analysis: POMWIG (implements diffraction into the HERWIG Monte Carlo Generator) B. Cox and J. Forshaw, Comput. Phys. Commun. 144, 104 (2002); DPEMC (extends POMWIG to include inclusive and exclusive DPE) M. Boonekamp and T. Kucs, Comput. Phys. Commun. 167, 217 (2005); ExHuME (implements the exclusive dijet production calculation of Ref. [9]), J. Monk and A. Pilkington, e-Print Archive: hep-ph/0502077. 